\title{
Effects of Morinda officinalis Polysaccharides on the Proliferation and Viability of Osteoblasts in Rats with Osteoporosis
}

\author{
F. QIAO, HANQING LI AND Y. X. TONG²*
}

Department of Orthopedics, Huaibei Miners General Hospital, Huaibei 235000, Anhui Province, ${ }^{1}$ Department of Orthopedics, Southwest Hospital, The Army Medical University, Chongqing 400038, ²Department of Orthopedics, The Second Affiliated Hospital of Inner Mongolia Medical University, Inner Mongolia Autonomous Region, Hohhot 010010, China

Qiao et al.: Effects of Morinda officinalis Polysaccharides in Rats

\begin{abstract}
We aimed to evaluate the effects of Morinda officinalis polysaccharides on the proliferation and viability of osteoblasts in rats with osteoporosis. 24 non-parous Sprague-Dawley rats were assigned into normal, model, low-dose and high-dose Morinda officinalis polysaccharide groups. The model of osteoporosis was established by ovariectomy. After successful modeling, $50 \mathrm{mg} / \mathrm{kg}$ and $75 \mathrm{mg} / \mathrm{kg}$ Morinda officinalis polysaccharides were subcutaneously injected in low-dose and high-dose Morinda officinalis polysaccharide groups, respectively. After $3 \mathrm{w}$ of intervention, osteoblasts were prepared using the skull and their proliferation index and alkaline phosphatase activity were measured. The histopathological changes in femoral tissues were observed. The bone mineral density and levels of serum osteocalcin, calcium and phosphorus, as well as the protein levels of receptor activator of nuclear factor kappa-B, receptor activator of nuclear factor kappa-B ligand and osteoprotegerin were determined. The proliferation index and alkaline phosphatase activity were highest in high-dose Morinda officinalis polysaccharide group ( $\mathbf{p}<0.05)$. Bone tissues had decreased cell spaces and gradually reduced spaces between the medullary cavities in Morinda officinalis polysaccharide groups. Model group had the highest bone mineral density and levels of serum osteocalcin, calcium and phosphorus $(\mathrm{p}<0.05)$. The protein expression levels of receptor activator of nuclear factor kappa-B ligand and receptor activator of nuclear factor kappa-B showed a decreasing trend, while that of osteoprotegerin displayed a rising trend sequentially in model, low-dose, high-dose Morinda officinalis polysaccharide and normal groups $(\mathbf{p}<\mathbf{0 . 0 5})$. Morinda officinalis polysaccharides promotes the proliferation of osteoblasts, enhances their viability and maintains the balance between bone resorption and formation in rats with osteoporosis probably by modulating the protein expression of the receptor activator of nuclear factor kappa-B ligand/receptor activator of nuclear factor kappa-B/osteoprotegerin pathway.
\end{abstract}

Key words: Osteoporosis, Morinda officinalis polysaccharides, osteoblasts, alkaline phosphatase, receptor activator of nuclear factor kappa-B

In recent years, the cases of osteoporosis, a systemic metabolic disease, have been increasing annually with the worsened aging. Osteoporosis is mainly characterized by osteopenia, degenerative changes in the microstructures of bone tissues and increased bone fragility ${ }^{[1]}$. A study showed that osteoblasts and osteoclasts are important players in the progression of osteoporosis and different degrees of apoptosis of osteoblasts are observed in various types of osteoporosis. Osteoblasts can facilitate the secretion of Osteoprotegerin (OPG) and Receptor Activator of Nuclear Factor Kappa-B Ligand (RANKL), thereby

*Address for correspondence E-mail: tongyxsahimmu@csc-edu.cn

January-February 2022 indirectly regulating the signal transduction of osteoclasts $^{[2]}$. Clinical medications for osteoporosis mainly repress bone resorption, but have no obvious effect on bone formation ${ }^{[3]}$.

In modern medicine, related traditional Chinese medicines are increasingly preferred for treating

\footnotetext{
This is an open access article distributed under the terms of the Creative Commons Attribution-NonCommercial-ShareAlike 3.0 License, which allows others to remix, tweak, and build upon the work non-commercially, as long as the author is credited and the new creations are licensed under the identical terms
}

Accepted 08 January 2022

Revised 03 June 2021

Received 26 November 2020 Indian J Pharm Sci 2022;84(1):58-65 
osteoporosis. Morinda officinalis (M. officinalis) is the dry root of the Rubiaceae plant. M. officinalis is originated from hybridization of two types of Rubiaceae plants, has the effects of invigorating the kidney, replenishing essence, strengthening the body and yang, and enhancing bones and muscles. It was found through the analysis of the therapeutically active ingredients of $M$. officinalis by modern medicine ${ }^{[4]}$ that beta $(\beta)$-anthraquinone, 2,4-diethyl cholesterol and amylose can effectively prevent tissue oxidation and protect the kidney to a certain degree. Besides, $M$. officinalis can stimulate the proliferation of osteoblasts cultured in vitro. A study corroborated that M. officinalis polysaccharides are one of the active ingredients to increase the viability of osteoblasts ${ }^{[5]}$. $M$. officinalis polysaccharides promote the differentiation of bone cells to a certain extent through affecting the expression of related proteins. According to the study where the fibroblasts were isolated from the hip joint capsular tissues in the patients with ankylosing spondylitis and femoral neck fracture and cultured, M. officinalis polysaccharides can repress the proliferation and osteogenic differentiation of normal fibroblasts and fibroblasts of patients with ankylosing spondylitis ${ }^{[6]}$. M. officinalis polysaccharides have significant efficacy in treating osteoporosis in the elderly and also speed up the proliferation of osteoblasts $^{[7]}$, but its mechanisms of action are rarely studied. The influences of $M$. officinalis polysaccharides on the proliferation and viability of osteoblasts in rats with osteoporosis and its mechanisms of action were explored in the present study. It is reported as follows.

\section{MATERIALS AND METHODS}

\section{Laboratory animals:}

A total of 24, 3 mo old specific pathogen-free grade non-parous Sprague-Dawley rats weighing $(210.1 \pm 5.4)$ $\mathrm{g}$ were provided by Beijing Vital River Laboratory Animal Technology Co., Ltd. They were fed using standard fodder in the environment with the room temperature of $(25 \pm 1)^{\circ}$, relative air humidity of $(52 \pm 2)$ $\%$ and ventilation of $8-12$ times $/ \mathrm{h}$. The experiments were conducted in this study with the approval from the ethics committee of our hospital.

\section{Main reagents:}

M. officinalis polysaccharides were sourced from Hangzhou MSD Pharmaceutical Co., Ltd., RANKL antibody was obtained from Boster Biological Technology Co., Ltd. (Wuhan, China) and Receptor
Activator of Nuclear Factor Kappa-B (RANK) was provided by Abcam (Shanghai) Trading Co., Ltd. OPG polyclonal antibodies were obtained from Abbkine Scientific Co., Ltd. and Phosphate Buffered SalineTween (PBST) was provided by Suzhou Yacoo Science Co., Ltd.

\section{Grouping and establishment of rat model of osteoporosis:}

6 out of 24 rats were selected as normal group and the remaining 18 rats were assigned into model group $(n=6)$, low-dose $M$. officinalis polysaccharide group $(\mathrm{n}=6)$ and high-dose $M$. officinalis polysaccharide group $(\mathrm{n}=6)$ using a random number table. Then, the rat model of osteoporosis was developed by the method described in the literature ${ }^{[8]}$. Specifically, at about $10 \mathrm{~min}$ after $10 \%$ chloral hydrate was intraperitoneally injected at $0.3 \mathrm{ml} / 100 \mathrm{~g}$, the rats had the four extremities fixed with the back upward when they had obviously weakened activity and even breath and no resistance when the four extremities were touched. Then, the hair of the back was removed and the back was conventionally disinfected using iodophor that was eluted using alcohol cotton balls later. Subsequently, small incisions (about $3 \mathrm{~mm}$ ) were separately made symmetrically in the left and right from the horizontal position of the costovertebral angle along the place that was parallel to the median waist and around $2 \mathrm{~mm}$ away from the midline. After the muscle was cut open, the retroperitoneum was accessed and the ovary was pinpointed along the uterine branch. Thereafter, the ovarian artery and vein were ligated at the fallopian tube, followed by dorsal ovariectomy. Afterwards, the muscle and skin were successively sutured and disinfected using iodophor. After fully awaking, the rats were returned to the cage and the modeling was observed. Obviously less movement in rats and fatigue, spasm and fractures due to the equal external force in the model rats suggested successful modeling. Successful modeling was observed in 15 rats which were divided into model group $(\mathrm{n}=5)$, low-dose M. officinalis polysaccharide group $(\mathrm{n}=5)$ and highdose M. officinalis polysaccharide group $(\mathrm{n}=5)$.

\section{Intervention methods:}

After successful modeling, $50 \mathrm{mg} / \mathrm{kg}$ and $75 \mathrm{mg} / \mathrm{kg}$ M. officinalis polysaccharides were subcutaneously injected for intervention in low-dose $M$. officinalis polysaccharide group and high-dose $M$. officinalis polysaccharide group, respectively, while an equal volume of normal saline was subcutaneously injected 
for intervention in normal group and model group. The intervention was conducted for $3 \mathrm{w}(1 \mathrm{~d} /$ time $)$ in the four groups, during which the rats were normally fed with free access to water.

\section{Culture of osteoblasts:}

After $3 \mathrm{w}$ of intervention, the rats were sacrificed by decapitation and soaked in $75 \%$ alcohol for $10 \mathrm{~min}$. Then, the scalp was removed aseptically. The skull was taken and the connective tissues on the surface were cleared. Next, the skull was rinsed using normal saline until it got blanched. After that, the skull was sheared into pieces and digested with $5 \mathrm{ml}$ of $0.25 \%$ trypsin for $20 \mathrm{~min}$. Thereafter, the digestive juice was discarded and the sclerite was digested for $60 \mathrm{~min}$ in the digestive juice containing $0.1 \%$ type I collagenase. Afterwards, the digestive juice was removed and the resulting sclerite was digested again in $0.1 \%$ type I trypsin for $60 \mathrm{~min}$. The filtered digestive juice was centrifuged at $2000 \mathrm{r} / \mathrm{min}$ for $5 \mathrm{~min}$, the supernatant was removed and the precipitated cells were added into $5 \mathrm{ml}$ of Dulbecco's Modified Eagle Medium (DMEM). Then, the cells were inoculated in a petri dish and cultured for $1 \mathrm{~d}$ in a $5 \%$ Carbon Dioxide $\left(\mathrm{CO}_{2}\right)$ incubator at $37^{\circ}$ with $95 \%$ humidity, followed by change of DMEM once. Thereafter, the non-adherent cells were removed and the medium was replaced once every 2-3 d. Finally, osteoblasts were purified and cultured until passage 3 for later use.

\section{Detection of proliferation of osteoblasts using MTT assay:}

The purified passage 3 osteoblasts were sub-cultured once and then they were digested by $0.25 \%$ trypsin and $0.02 \%$ ethylenediaminetetraacetic acid and counted. After that, the cells were seeded into a 96-well plate at a density of $2 \times 10^{3}$ cells/well and cultured for $24 \mathrm{~h}$ with $5 \mathrm{ml}$ of DMEM containing $10 \%$ calf serum in the environment with $5 \% \mathrm{CO}_{2}$ at $37^{\circ}$. Upon stable cell adhesion, the cells were washed using balanced salt solution and the proliferation of osteoblasts was detected by the 3-(4,5-Dimethylthiazol-2-yl)-2,5Diphenyl Tetrazolium Bromide (MTT) assay using the reagent (Batch No. BJ-0251BT, Shanghai Bangjing Industrial Co., Ltd.). Finally, the Absorbance (A) value was read using a microplate reader (model No. MR-96A, Nanjing Vedeng Medical Co., Ltd.) at the wavelength of $490 \mathrm{~nm}$ and the proliferation index of osteoblasts was analyzed.

\section{Determination of Alkaline Phosphatase (ALP) activity in osteoblasts using para-nitrophenyl phosphate assay:}

The cells were cultured as described above. The ALP activity in osteoblasts was analyzed by the paranitrophenyl phosphate assay using the reagent (batch No. S46070-1 g, Shanghai Yanjin Biological Co., Ltd.) and the A value was read using the microplate reader at the wavelength of $405 \mathrm{~nm}$, followed by analysis of the ALP activity in osteoblasts.

\section{Pathological observation:}

The femoral bone tissues were quickly intercepted from the sacrificed rats in each group, fixed in $4 \%$ paraformaldehyde and conventionally prepared into paraffin-embedded sections. Then, the sections were stained in $0.5 \%$ hematoxylin-eosin staining solution for $10 \mathrm{~min}$, rinsed using tap water once and dehydrated in gradient ethanol. Finally, the resulting sections were sealed by neutral resin.

\section{Detection of bone metabolism-related indicators:}

Before killing the rats, $3 \mathrm{ml}$ of abdominal venous blood was drawn and centrifuged at $3000 \mathrm{r} / \mathrm{min}$ for $10 \mathrm{~min}$ and the upper serum was isolated for the detection of the following bone metabolism-related indicators.

Level of osteocalcin: First, the antigens were dissolved to $10-20 \mu \mathrm{g} / \mathrm{ml}$ using $5 \mathrm{mmol} / 1$ carbonate coating buffer, added into a 96-well enzyme-linked immunosorbent assay plate at $100 \mu \mathrm{l} /$ well and stored at $4^{\circ}$ overnight. The next day, the coating solution was discarded and the resulting antigens were washed using PBST three times, added with $150 \mu$ of $1 \%$ bovine serum albumin (article No. LM-161, Shanghai Lianmai Biological Engineering Co. Ltd.) per well and sealed at $37^{\circ}$ for $1 \mathrm{~h}$. After that, the mixture was washed using PBST three times and each well was added with $100 \mu$ of bovine serum albumin diluted at different ratios and osteocalcin standard (Shanghai J\&L Biological Co., Ltd.), followed by incubation at $37^{\circ}$ for $2 \mathrm{~h}$. After being washed with PBST five times, the products were incubated with 100 $\mu 1$ of horseradish peroxidase (Shanghai Yuanye BioTechnology Co., Ltd.) labeled secondary antibodies diluted at 1:5000 (batch No. YM-XQ0470P, Yuanmu Biotechnology Co., Ltd.) at $37^{\circ}$ for $1 \mathrm{~h}$. The resulting products were washed again with PBST five times and developed using the color developing agent for $20 \mathrm{~min}$. Finally, the A value was measured using the microplate reader at $405 \mathrm{~nm}$ to determine the level of osteocalcin. 
Level of calcium: The level of calcium was measured strictly according to the instructions of the Arsenazo III kit and related reagents (Beijing Simes-sikma Biotech Co., Ltd.).

Level of phosphorus: A total of $2 \mathrm{ml}$ of venous blood was collected into a $50 \mathrm{ml}$ digestion tube and added with $15 \mathrm{ml}$ of mixed acid, followed by standing overnight. On the $2^{\text {nd }} \mathrm{d}$, the digestion tube was placed in the digestion furnace. At the beginning of the digestion, the temperature could be turned down by about $130^{\circ}$ and then gradually up to $240^{\circ}$ for digestion until white smoke was observed or the liquid became colorless or yellowish-green. A total of $2 \mathrm{ml}$ each of sample and empty solution was added into a $20 \mathrm{ml}$ test tube with stopper, followed by addition of $2 \mathrm{ml}$ of ammonium molybdate solution, $1 \mathrm{ml}$ of sodium sulfite solution and $1 \mathrm{ml}$ of hydroquinone solution successively. After that, the mixture was added with distilled water to the constant volume of $20 \mathrm{ml}$, mixed well and let stand for $30 \mathrm{~min}$. Finally, the A value was determined at $660 \mathrm{~nm}$, by which the content of phosphorus was calculated in the standard curve.

Bone mineral density: The femoral bone mineral density of the rats was measured using a dual-energy $\mathrm{X}$-ray bone densitometer.

Measurement of protein expression levels of RANKL, RANK and OPG via western blotting:

After $3 \mathrm{w}$ of intervention, the extracted osteoblast cells of the rats were ground and added with protein buffer for conventionally extracting protein. Then, the total protein was quantitatively analyzed using the reagent (batch No. B4509, Beijing Puxi Technology Co., Ltd.) by the bicinchoninic acid method. A total of $50 \mu \mathrm{g}$ of protein samples were loaded and then subjected to sodium lauryl sulfate-polyacrylamide gel electrophoresis. Subsequently, the proteins were electrotransferred onto a polyvinylidene fluoride membrane and sealed in TrisBuffered Saline with Tween-20 (TBST) containing 5 $\%$ skim milk powder (batch No. H0013, Suzhou Yacoo Science Co., Ltd.) without light for $1 \mathrm{~h}$. After being washed, the proteins were added with the diluents of the primary antibodies against RANKL, RANK and OPG (1:1000) and stored at $4^{\circ}$ overnight. The resulting proteins were rinsed using TBST on the next day, added with the diluents of the secondary antibodies (1:5000) and incubated in a hotbed for $1 \mathrm{~h}$. After being washed again using TBST, the proteins were added with electrochemiluminescence reagent (Biochannel) and exposed 2-3 times. Finally, the average value was obtained. The gray value was analyzed using ImageLab software and the relative molecular mass of proteins was calculated with glyceraldehyde-3-phosphate dehydrogenase as an internal reference. The antibodies were purchased from Shanghai Shixi Chemical Technology Co., Ltd.

\section{Statistical analysis:}

Statistical Package for the Social Sciences (SPSS) 26.0 software was used for statistical analysis. Measurement data were expressed as $(\overline{\mathrm{x}} \pm \mathrm{s})$. Analysis of variance was performed for comparisons among multiple groups and Least Significant Difference (LSD)-t test was adopted for pairwise comparisons. $\mathrm{p}<0.05$ was considered statistically significant.

\section{RESULTS AND DISCUSSION}

The proliferation index and ALP activity were the highest in high-dose $M$. officinalis polysaccharide group, followed by those in low-dose M. officinalis polysaccharide group, normal group and model group $(\mathrm{p}<0.05)$ (fig. 1).

Pathological changes in femoral tissues were given here. Normal group had regularly and orderly distributed cells in the bone tissues, with smaller spaces. In model group, the bone tissues had larger spaces between the medullary cavities and the cells were irregularly and loosely distributed. The bone tissues had decreased cell spaces and gradually reduced spaces between the medullary cavities in $M$. officinalis polysaccharide groups, especially in high-dose $M$. officinalis polysaccharide group (fig. 2).

Results of bone metabolism-related indicators are shown here. Model group had the highest bone mineral density and level of serum osteocalcin, followed by low-dose $M$. officinalis polysaccharide group, highdose $M$. officinalis polysaccharide group and normal group, and the highest levels of serum calcium and phosphorus, followed by low-dose $M$. officinalis polysaccharide group, normal group and high-dose M. officinalis polysaccharide group $(\mathrm{p}<0.05)$ (fig. 3).

The relative protein expression levels of RANKL and RANK showed a decreasing trend, whereas the relative protein expression level of OPG exhibited a rising trend successively in model group, low-dose M. officinalis polysaccharide group, high-dose $M$. officinalis polysaccharide group and normal group $(\mathrm{p}<0.05)$ (fig. 4). 
A

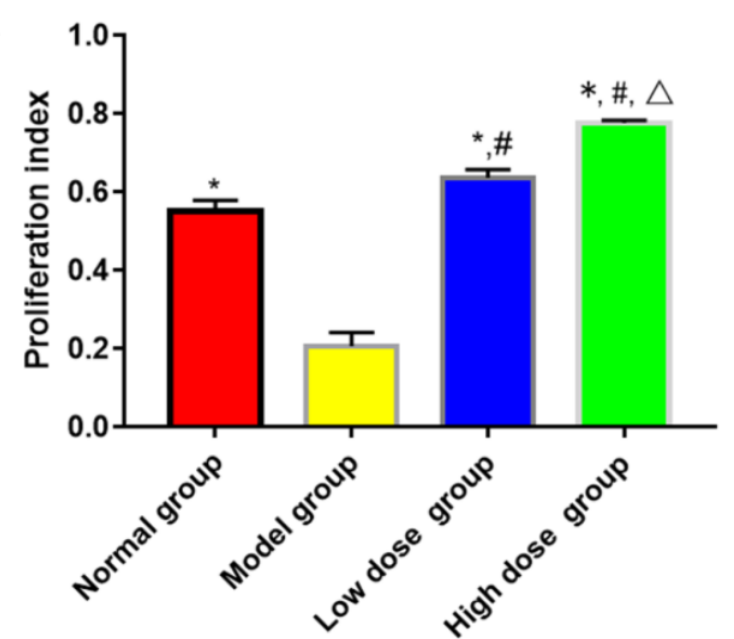

B

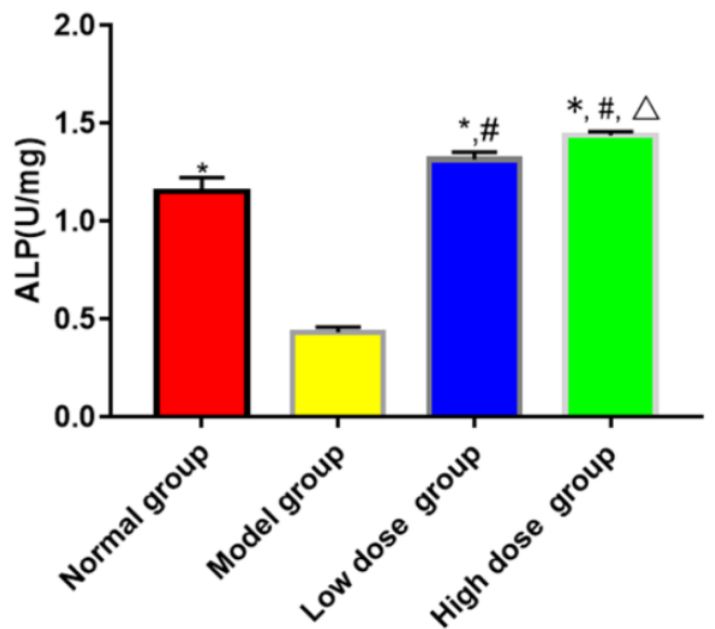

Fig. 1: Proliferation index and ALP activity of osteoblasts, ${ }^{*} \mathbf{p}<0.05$ vs. model group, ${ }^{\#} \mathbf{p}<0.05$ vs. normal group, ${ }^{\wedge} \mathbf{p}<0.05$ vs. low-dose M. officinalis polysaccharide group
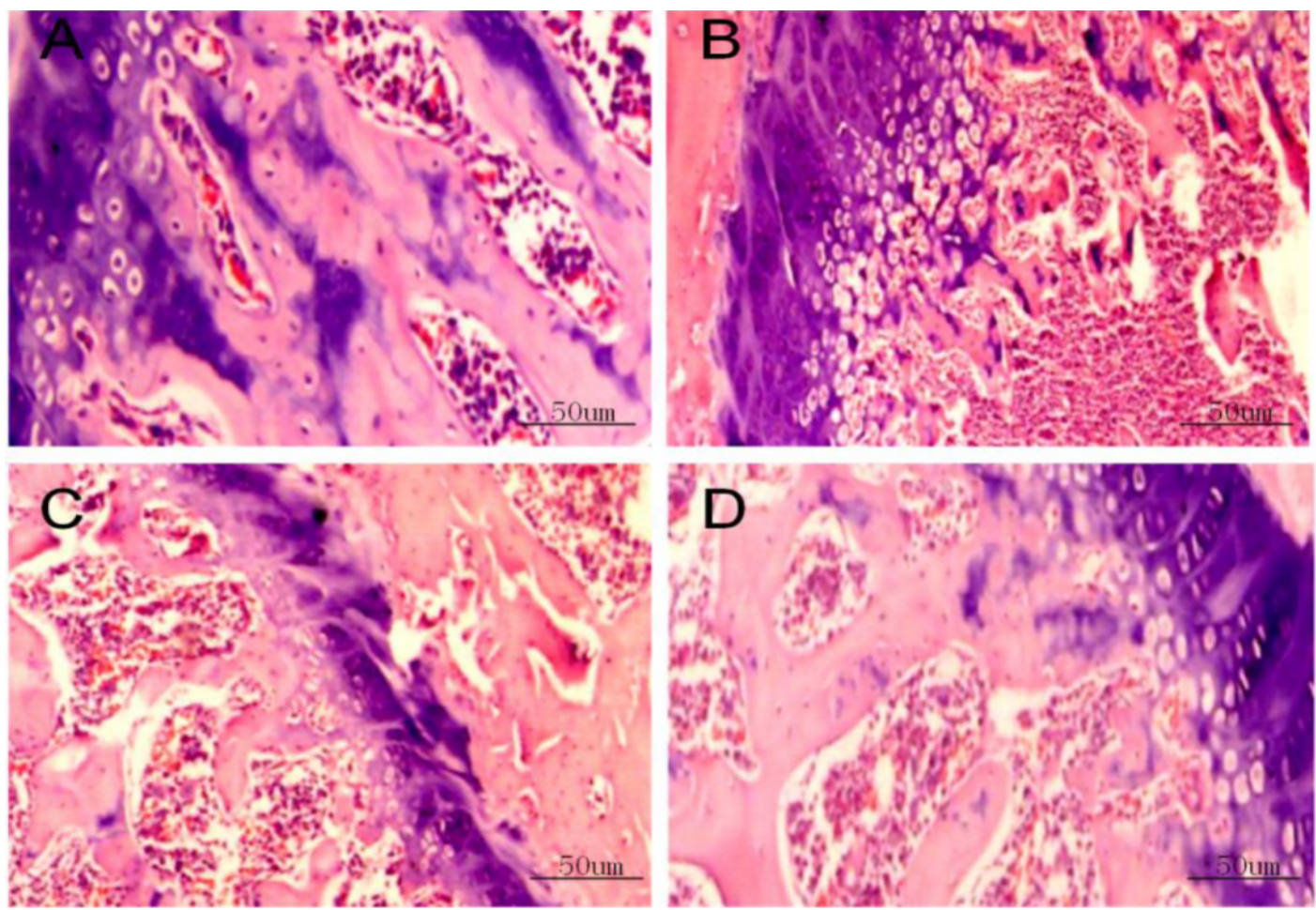

Fig. 2: Pathological changes in femoral tissues of rats in each group detected by hematoxylin and eosin staining $(\times 400)$. (A) Normal group; (B) Model group; (C) Low-dose M. officinalis polysaccharide group; (D) High-dose M. officinalis polysaccharide group

Inbonemetabolism, osteoclastspromoteboneresorption, while osteoblasts facilitate bone formation and the normal bone mass of the body can be maintained as long as bone absorption and bone formation are relatively balanced ${ }^{[9]}$. Pancreatic islet function gradually declines with the increase in age and the risk of osteoporosis is also gradually heightened. Osteoporosis is much likely to cause disability, seriously affecting the quality of life of people. M. officinalis polysaccharides have similar chemical structures to estrogens, so they can play a role as estrogens and yield significant efficacy in preventing postmenopausal osteoporosis with few toxic and side effects $^{[10]}$. In the present study, the histopathological results showed that the rats in model group had larger spaces between the medullary cavities of the bone tissues and irregularly and loosely distributed cells in the bone tissues, suggesting that the model was successfully established. ALP, one of the major enzymes in bone formation, can reflect the differentiation, maturity and viability of osteoblasts and its activity is gradually enhanced as osteoblasts differentiate while reflecting the viability of osteoblasts ${ }^{[11,12]}$. Besides, ALP is a vital marker for bone matrix formation, which is able to reflect bone formation and evaluate bone 
A

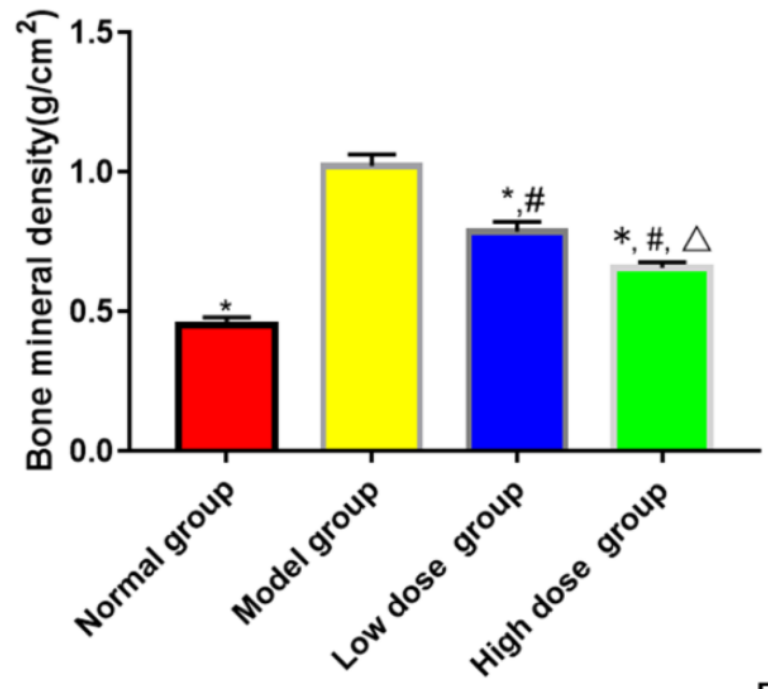

C

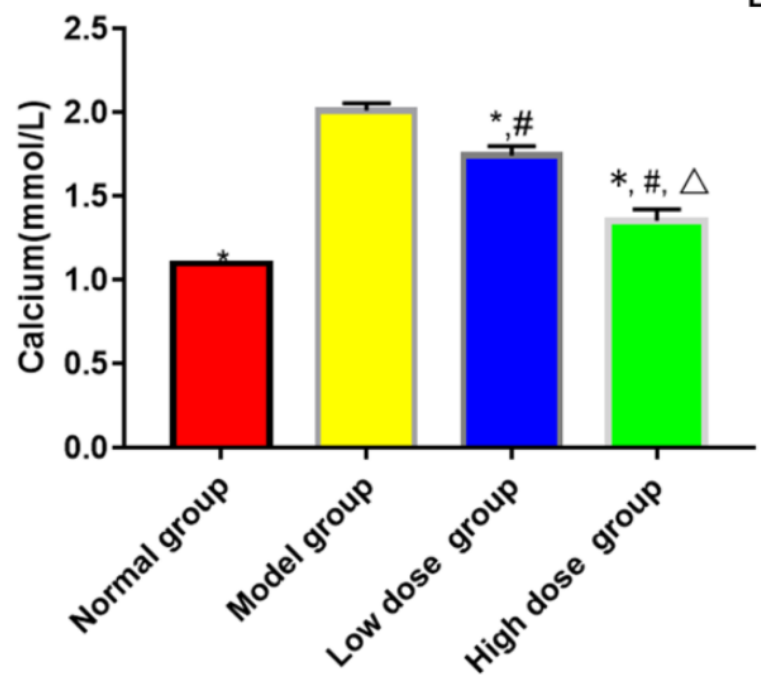

B
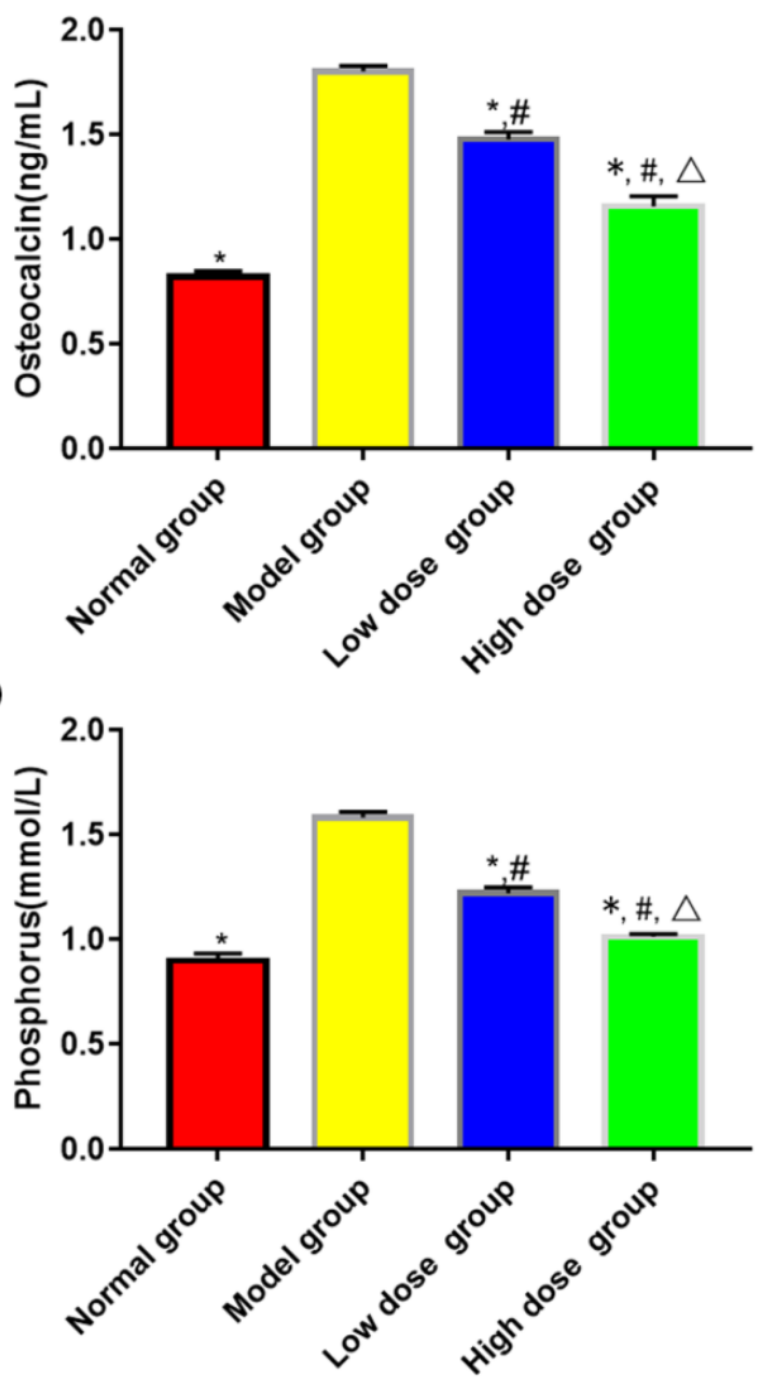

Fig. 3: Comparative bone metabolism-related indicators in the four groups of rats. (A) Bone mineral density; (B) Osteocalcin; (C) Calcium; (D) Phosphorus, * ${ }^{*}<0.05$ vs. model group, ${ }^{\#} \mathbf{p}<0.05$ vs. normal group, ${ }^{\Delta} \mathbf{p}<0.05$ vs. low-dose $M$. officinalis polysaccharide group

A

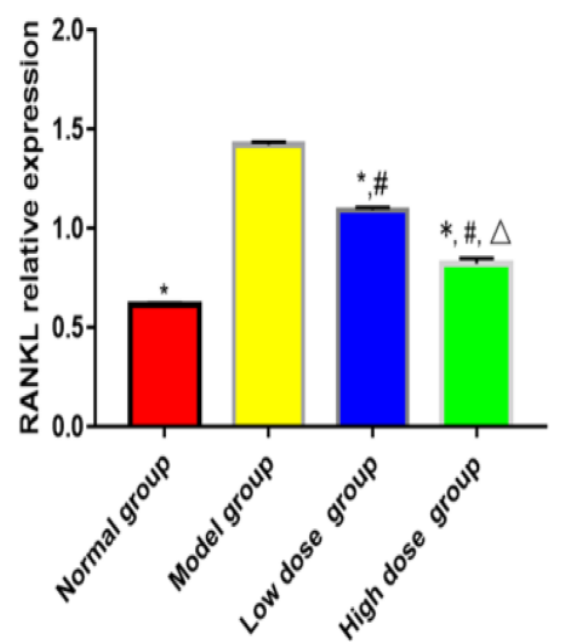

B

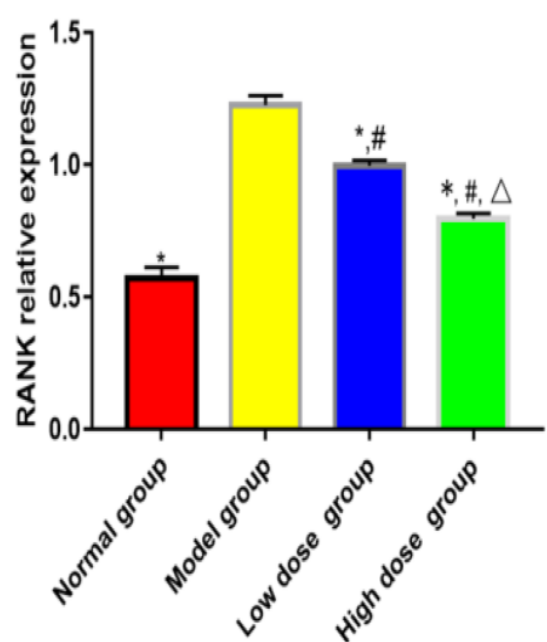

C

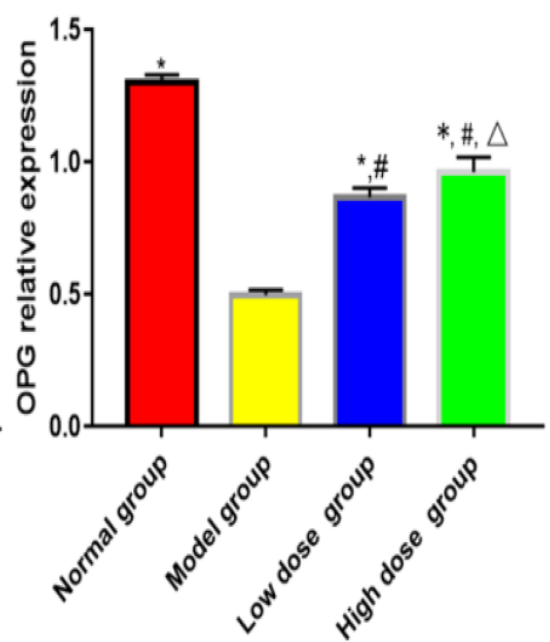

Fig. 4: Relative protein expression levels of (A) RANKL; (B) RANK and (C) OPG in the four groups of rats, *p<0.05 vs. model group, ${ }^{\#} \mathbf{p}<0.05$ vs. normal group, ${ }^{\Delta} \mathbf{p}<0.05$ vs. low-dose $M$. officinalis polysaccharide group 
transformation ${ }^{[13]}$. According to the results of this study, low-dose and high-dose $M$. officinalis polysaccharide groups had higher proliferation index and ALP activity of osteoblasts than model group and normal group $(\mathrm{p}<0.05)$, indicating that $M$. officinalis polysaccharides can enhance the viability of osteoblasts. Moreover, the proliferation index and ALP activity in highdose $M$. officinalis polysaccharide group were higher than those in low-dose $M$. officinalis polysaccharide group $(\mathrm{p}<0.05)$, implying that high-dose $(75 \mathrm{mg} / \mathrm{kg})$ $M$. officinalis polysaccharides are better in increasing ALP activity and have a more significant inducing effect on the proliferation of osteoblasts. A study demonstrated that the increase in bone metabolism markers suggests the accelerated bone transformation and bone $\operatorname{loss}^{[14]}$. Serum calcium and phosphorus are the important bone mineral compositions and their stable content is the essential sign of balance between bone resorption and bone formation ${ }^{[15]}$. In this study, it was found that bone mineral density and levels of osteocalcin, calcium and phosphorus in the serum of rats in model group were higher than those in the other three groups $(p<0.05)$, indicating that osteoporosis can speed up bone transformation and bone loss. The bone mineral density and levels of osteocalcin, calcium and phosphorus in model group were the highest, followed by those in low-dose $M$. officinalis polysaccharide group and highdose $M$. officinalis polysaccharide group $(\mathrm{p}<0.05)$. This suggests that $M$. officinalis polysaccharides can reduce bone mineral density and serum osteocalcin, calcium and phosphorus content in rats and maintain the balance between bone resorption and bone formation and highdose $(75 \mathrm{mg} / \mathrm{kg})$ M. officinalis polysaccharides have a more significant effect.

The RANKL/RANK/OPG pathway is an important regulatory pathway in bone resorption and bone formation, where OPG protein, the only receptor binding to RANKL on the membrane of osteoblasts, mainly works to inhibit the binding between RANKL and RANK, thereby blocking the differentiation and fusion of osteoclast precursors and ultimately suppressing mature osteoclasts and repressing the apoptosis of osteoblasts to a certain extent ${ }^{[16,17]}$. After being stimulated by bone resorption, osteoblasts are capable of promoting the secretion of RANKL that can bind to RANK on the surface of osteoclast precursor cells and activating the nuclear factor kappa B pathway, thereby facilitating the differentiation and formation of mature osteoclasts ${ }^{[18,19]}$. After being stimulated by osteogenesis-related factors, osteoblasts can induce the secretion of OPG that will compete with RANKL to bind to RANK, thus repressing the formation and viability of osteoclasts. The results of this study revealed that the relative expression levels of RANKL and RANK were the highest in model group, followed by those in lowdose $M$. officinalis polysaccharide group and high-dose M. officinalis polysaccharide group, while the relative expression level of OPG was the highest in highdose $M$. officinalis polysaccharide group, followed by that in low-dose M. officinalis polysaccharide group and model group $(\mathrm{p}<0.05)$. These results imply that M. officinalis polysaccharides may enhance the viability of osteoblasts in rats with osteoporosis by regulating the RANKL/RANK/OPG pathway.

In conclusion, $M$. officinalis polysaccharides are able to promote the proliferation and enhance the viability of osteoblasts and maintain the balance between bone resorption and bone formation in rats with osteoporosis probably through modulating the protein expression of the RANKL/RANK/OPG pathway.

\section{Author's contributions:}

Feng Qiao and Hanqing Li contributed equally to this work.

\section{Conflict of interests:}

The authors declared no conflict of interest.

\section{REFERENCES}

1. Scholten DJ, Bray JK, Wang KY, Lake AF, Emory CL. Implementation of a fracture liaison service and its effects on osteoporosis treatment adherence and secondary fracture at a tertiary care academic health system. Arch Osteoporos 2020;15:1-4.

2. Bai P, Sun Y, Jin J, Hou J, Li R, Zhang Q, et al. Disturbance of the OPG/RANK/RANKL pathway and systemic inflammation in COPD patients with emphysema and osteoporosis. Respir Res 2011;12(1):1-8.

3. Zhou L, Liu Q, Hong G, Song F, Zhao J, Yuan J, et al. Cumambrin A prevents OVX-induced osteoporosis via the inhibition of osteoclastogenesis, bone resorption and RANKL signaling pathways. FASEB J 2019;33(6):6726-35.

4. Liu ZW, Luo ZH, Meng QQ, Zhong PC, Hu YJ, Shen XL. Network pharmacology-based investigation on the mechanisms of action of Morinda officinalis How. in the treatment of osteoporosis. Comput Biol Med 2020;127:104074.

5. Zhang D, Zhang S, Jiang K, Li T, Yan C. Bioassay-guided isolation and evaluation of anti-osteoporotic polysaccharides from Morinda officinalis. J Ethnopharmacol 2020;261:113113.

6. Ye LI, Shan-Shan LÜ, Gui-Ying TA, Min HO, Qing TA, Zhang $\mathrm{XN}$, et al. Effect of Morinda officinalis capsule on osteoporosis in ovariectomized rats. Chin J Nat Med 2014;12(3):204-12.

7. MengYong Z, CaiJiao W, HuSheng Z, XianWu P, JianMin F. Protective effect of polysaccharides from Morinda officinalis on bone loss in ovariectomized rats. Int J Biol Macromol 2008;43(3):276-8.

8. Zhang HS, Li T, Liu XS, Wang FC, Yao X, Yan L, et al. 
Experimental study of the effect on bone metabolism and bone histomorphometry of osteoporosis rats with birdpecking and revolving moxibustion on twelve back-shu points. Cell Biochem Biophys 2015;71(1):173-8.

9. Lenora J, Ivaska KK, Gerdhem P. Use of bone turnover markers in osteoporosis. Clin Rev Bone Miner Meta 2010;8(1):1-14.

10. Zhu Z, Zhao X, Huang F, Wang F, Wang W. Morinda officinalis polysaccharides attenuate varicocele-induced spermatogenic impairment through the modulation of angiogenesis and relative factors. Evid Based Complement Alternat Med 2019;2019.

11. Chou LY, Chen CH, Chuang SC, Cheng TL, Lin YH, Chou $\mathrm{HC}$, et al. Discoidin domain receptor 1 regulates runx2 during osteogenesis of osteoblasts and promotes bone ossification via phosphorylation of p38. Int J Mol Sci 2020;21(19):7210.

12. Qiu WX, Ma XL, Lin X, Zhao F, Li DJ, Chen ZH, et al. Deficiency of Macfl in osterix expressing cells decreases bone formation by Bmp2/Smad/Runx2 pathway. J Cell Mol Med 2020;24(1):317-27.

13. Bernhardt A, Österreich V, Gelinsky M. Three-dimensional co-culture of primary human osteocytes and mature human osteoclasts in collagen gels. Tissue Eng Part A 2020;26(1112):647-55.

14. Ren Q, Wu Y, Ma J, Shan Q, Liu S, Liu Y. Carbon black- induced detrimental effect on osteoblasts at low concentrations: Remarkably compromised differentiation without significant cytotoxicity. Ecotoxicol Environ Saf 2019;178:211-20.

15. Yin Y, Ding L, Hou Y, Jiang H, Zhang J, Dai Z, et al. Upregulating MicroRNA-410 or downregulating Wnt-11 increases osteoblasts and reduces osteoclasts to alleviate osteonecrosis of the femoral head. Nanoscale Res Lett 2019;14(1):1-6.

16. Doustimotlagh AH, Dehpour AR, Etemad-Moghadam S, Alaeddini M, Ostadhadi S, Golestani A. A study on OPG/ RANK/RANKL axis in osteoporotic bile duct-ligated rats and the involvement of nitrergic and opioidergic systems. Res Pharm Sci 2018;13(3):239-49.

17. Kim JY, Kim HJ, Kim CS. Effects of 12-week combined exercise on RANKL/RANK/OPG signaling and boneresorption cytokines in healthy college females. J Exerc Nutrition Biochem 2019;23(1):13-20.

18. Liu Y, Xu Z, Wang Q, Jiang Y, Wang R, Chen S, et al. Selective regulation of RANKL/RANK/OPG pathway by heparin sulfate through the binding with estrogen receptor $\beta$ in MC3T3-E1 cells. Int J Biol Macromol 2020;161:1526-34.

19. Amin N, Boccardi V, Taghizadeh M, Jafarnejad S. Probiotics and bone disorders: The role of RANKL/RANK/OPG pathway. Aging Clin Exp Res 2020;32(3):363-71. 